\title{
Recent upgrades to the nuclear data tool JANIS
}

\author{
Nicolas Soppera, Manuel Bossant, Hans Henriksson, Pierre Nagel, and Yolanda Rugama \\ OECD Nuclear Energy Agency, 12 boulevard des Îles, 92130 Issy-les-Moulineaux, France
}

\begin{abstract}
JANIS (JAva-based Nuclear Information Software) is a display program designed to facilitate the visualisation and manipulation of nuclear data. Its objective is to allow the user of nuclear data to access numerical and graphical representations without prior knowledge of the storage format. Several standardised data formats are supported, and data originating from the major evaluation libraries, such as ENDF/B, JEFF, JENDL, BROND, etc., can be displayed, inter-correlated, and compared with experimental reaction data in the database EXFOR. Various navigation tools are available to identify the nucleus of interest, and the properties of the selected nuclide are displayed using textual, graphical or tabular formats. JANIS includes the bibliographical database CINDA, where charged particle data has been added recently permitting to establish a link between CINDA and EXFOR. JANIS has developed into a popular tool among researchers and data evaluators and the number of users increase steadily. The program's database is queried over 25,000 times per month. Features included in the latest release are described, and examples given on the use of JANIS.
\end{abstract}

\section{Introduction}

JANIS is a display program for nuclear data developed by the Nuclear Energy Agency (NEA) [1]. It offers flexibility for the comparison of different nuclear data sets. Its powerful and user-friendly navigation tools make it also suitable for educational purposes. JANIS is free of charge.

The latest version of JANIS reads the standard EXFOR format and performs advanced manipulations of data, like calculating the product of cross sections with energy and angular distributions. Differential cross section data can be plotted and the output formats include vectorial graphics formats (PostScript, and now also Windows MetaFile, WMF). The program is integrated in the NEA web data retrieval services so that users easily can plot the results, and compare with other data in the NEA database, or with personal data, using the import feature of JANIS.

JANIS has become a popular tool among researchers and data evaluators and the number of users increase steadily. The program's database is accessed over 25,000 times per month. Features included in the latest release are described, and examples given on the use of JANIS.

Figure 1 shows the usage of JANIS and the access to the remote databases with the application. The different versions indicated illustrate important steps in the development. Version 2.1 was distributed at the last nuclear data conference, ND-2004 in Santa Fe, and is the most used version. JANIS was linked to the NEA web retrieval system in the end of 2005 with the release of JANIS-2.2.1.

\section{What's new in JANIS-3}

The main updates included in JANIS-3 are given below. The program contains the latest evaluated data libraries: JEFF3.1, ENDF/B-VII, JENDL-3.3 and FENDL-2.1. Emphasis has been put in including the complete EXFOR database [2] in the original format, previous versions were using a

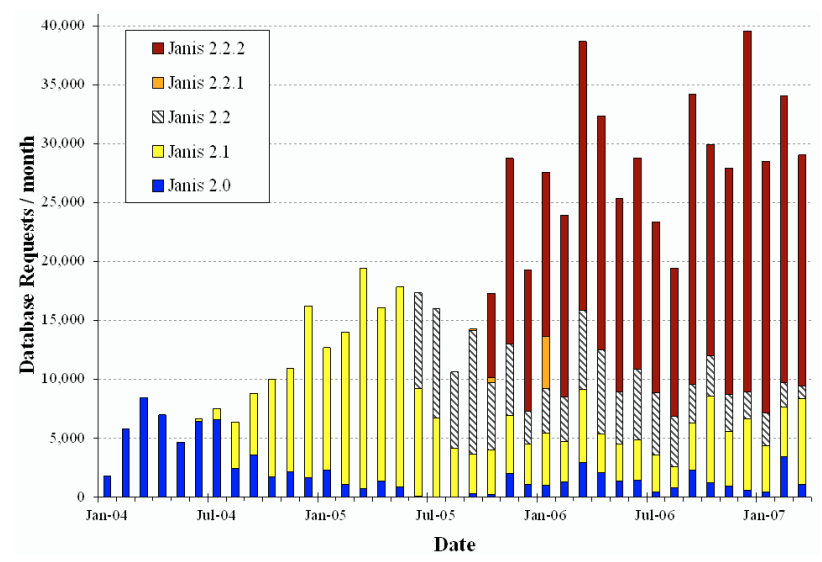

Fig. 1. Number of access to the JANIS database at the NEA each month, since the release of JANIS-2. Version 2.1 was distributed at the ND-2004 conference in Santa Fe, and JANIS-2.2.1 was the first version to be used with the NEA web retrieval pages. (Note that one user generates in general many requests while plotting data and searching the databases.)

simplified format, the EXFOR computational format, which only contains numerical values, and only for a subset of the EXFOR content. The user can now search for any data in the database, tabulate or plot most of what can be found, and display the whole textual information entered in EXFOR.

Bibliographical data in the upgraded CINDA database is also available, and new search interfaces for the EXFOR and CINDA databases have been developed.

Plotting was improved with vectorial rendering; this allows to change line widths and style, e.g., dashed lines, and to produce outputs (prints or files) without pixelisation. The user can customize all fields, e.g., titles, $\mathrm{x}$-axis unit presentation, colours, fonts, etc. JANIS export graphs in PNG and postscript (PS, EPS) and the possibility to export Windows Meta-File (WMF, EMF) files has now also been added, allowing easier insert in Office documents. 
JANIS is now integrated as the plotting tool for the EVA and EXFOR web retrieval pages. For further usage, see: www.nea.fr/html/dbdata/eva, www.nea.fr/html/dbdata/x4.

\section{Using JANIS}

JANIS is designed to be user-friendly, and straight-forward to use from the beginning. Advanced users can further improve the usability by using their preferred settings and specific databases. The program is a stand-alone application with direct access to large databases.

\subsection{Getting started}

This program is free of charge, and can be downloaded from the JANIS homepage, www.nea.fr/janis, where additional information can be found as well. Alternatively, users can request a DVD from the NEA (neapub@nea.fr.), which contains the software, databases and data files. JANIS is based entirely on Java so it is fully platform-independent and can be used on almost all computer operating systems, e.g., Windows, Linux and Mac OS X. Java is freely available at www.java.com.

The software can be installed like any other program as an independent application, but it can also be launched without pre-installation on the user's computer. If, for example, JANIS is launched via the EVA and EXFOR web retrieval systems, a plot window is displayed immediately thanks to a technology named Java Web Start (part of the Java installation) that launches applications like JANIS directly from an Internet page, ensuring that the latest version available will be automatically used.

JANIS accesses data from several different nuclear data libraries and bibliographic information from the CINDA database. The input formats supported are ENDF-6 [3] (along with the linearised point-wise option PENDF and the groupwise option GENDF), the original EXFOR format, as well as nuclear structure data from NUBASE [4].

\subsection{Types of data included}

The most common type of nuclear data is the particle interaction data. The user finds cross-sections (point-wise and multi-group data) with associated uncertainties, resonance parameters, energy distributions, angular distributions and correlated energy-angle distributions. Experimental data from EXFOR can be plotted as well, with advanced search options combining reaction identifiers, projectile energy range, laboratory, and experiment date. Bibliographic information from CINDA is retrievable, and is cross-correlated with EXFOR, as well as journal web pages for many of the references included in the database.

Radioactive decay data is included in JANIS. A summary of the important properties of radioactive nuclides is provided with mass of the nuclide, excitation energy, spin and parity, half-life, mean decay energies and decay modes. For each decay mode, the corresponding $\mathrm{Q}$ value, branching ratio and

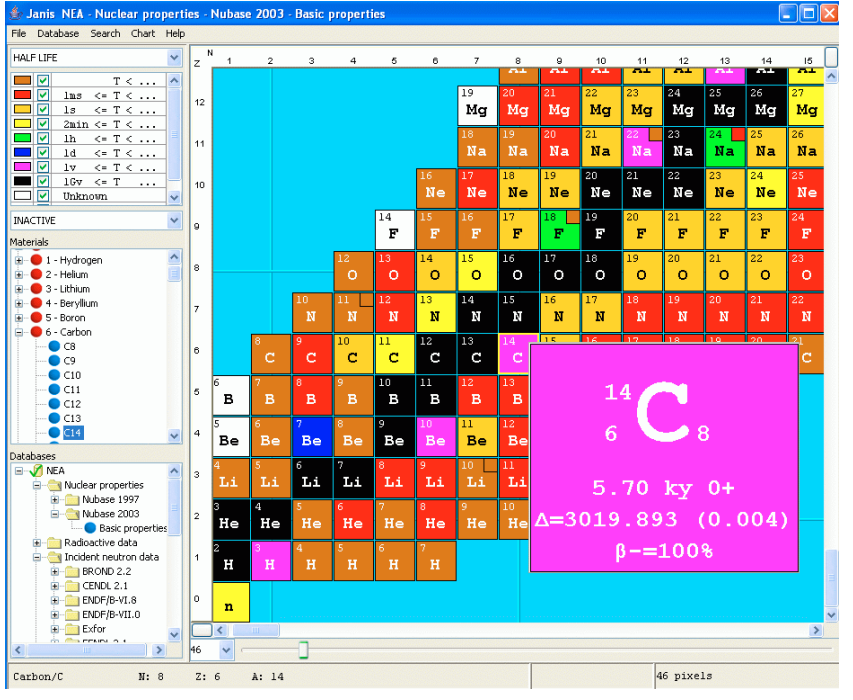

Fig. 2. The main browser window of JANIS. Nuclide ${ }^{14} \mathrm{C}$ has here been selected (see middle-left pane) from Nubase-2003 (see the bottom-left pane), and its basic properties are shown in the nuclide chart (see the right pane).

nuclide produced are given. The decay path of a particular nuclide can be tabulated and plotted. Discrete and continuous spectra of emitted particles ( $\gamma$ - and X-rays, $\alpha$-particles, $\beta^{+}$and $\beta^{-}$) are also available, such as energy of the emitted particle, relative and absolute line intensities and the associated uncertainties.

Fission yields are also available as independent and cumulative yields. Yields are given for typical neutron spectra (thermal neutron-induced, fast neutron-induced and highenergy neutron-induced fission). Spontaneous fission yields are provided as well.

\subsection{Browsing the nuclide chart}

When JANIS is launched, the browser window appears. This window contains a "chart of nuclides" pane with the isotopes that are available in a certain database selected in the bottomleft pane. The middle-left pane contains a list of the isotopes, and the top-left pane shows the legend of the chart pane. The bar at the bottom of the window is used to zoom. By zooming or right-clicking on an isotope, further data on that isotope is shown. An example of a nuclide chart (Nubase 2003) is shown in figure 2, where the user has right-clicked on ${ }^{14} \mathrm{C}$. By doubleclicking on an isotope, the plot window appears. (Ctrl-G can be used to find the isotope of interest.)

\subsection{Plotting facilities}

The plot window contains three main parts, namely, the data pane with graphs, tabular data or decay paths (shown in different "tabs"), the settings pane (to the right) used to set the $\mathrm{x}$ - and $\mathrm{y}$-axes, zoom, titles, colours, unit format, etc., the selection pane in the bottom of the window is used to choose what data to handle (see also fig. 3 for an example of a 


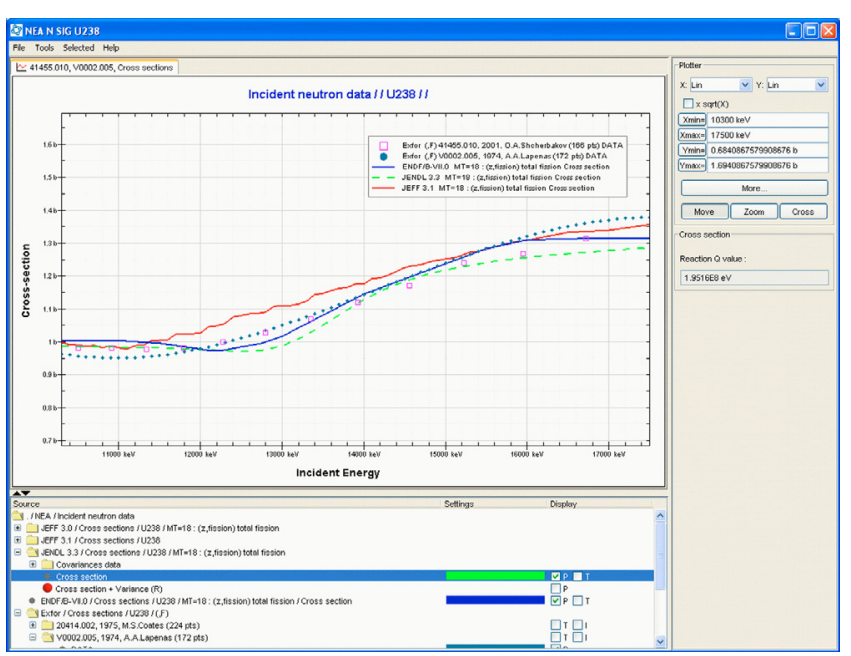

Fig. 3. The plot window, with evaluated and experimental fission cross section data on ${ }^{238} \mathrm{U}$ displayed together. The selected data is shown in the bottom part of the figure. The settings of the plot are available on the right-hand side.

comparison between experimental and evaluated fission cross section data on ${ }^{238} \mathrm{U}$ at high energies).

The selection pane offers several checkboxes for each data set, allowing the user to choose what should be displayed and in which for. Plots are viewed with the option "P", and tabular data with "T". Some data is displayed as text with the option "I", and " $D$ " is used for decay paths.

Right-clicking on a selected data set gives a menu of choices for manipulating or comparing the data set. See also the menu bar for more options.

\subsection{Comparing and searching}

There are many possibilities to search in the databases included in JANIS. The main menu has the "Search" option, where all possible search dialogs are listed:

- The EXFOR search window contains most keywords that are included in the EXFOR format.

- CINDA includes searches on the reaction string as well as a cross correlation with EXFOR data.

- The ENDF search window.

- Decay lines given in ENDF libraries.

- Resonances given in ENDF libraries.

The user can also access the search dialogs by right-clicking on the selected data set in the bottom pane of the plot window, and choose "search". This actually is a fast way to compare data, as the same type of reaction is pre-filled in and the search automatically launched, so that the relevant search results appear immediately. The results can of course be further refined by the user. Figure 4 shows the EXFOR search dialog, where for example several keywords used in the bibliographic section of the EXFOR entry can be used to filter the results.

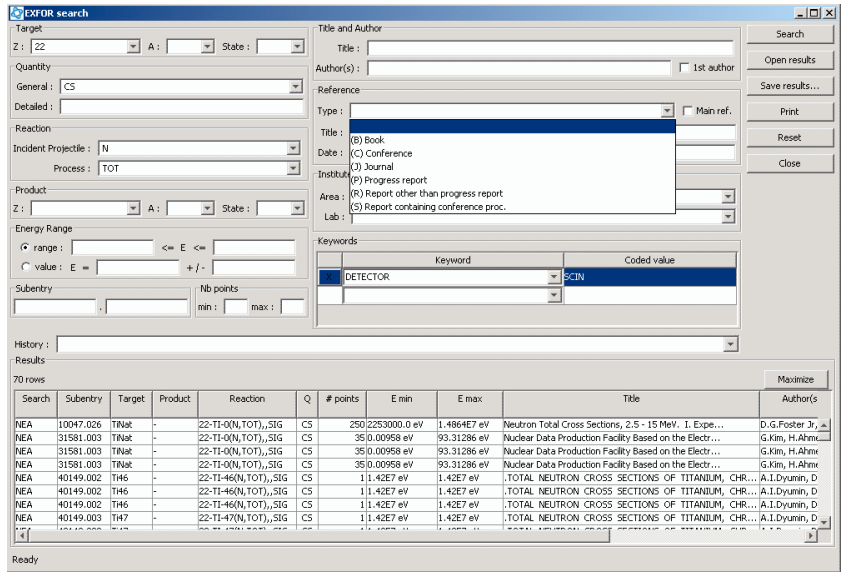

Fig. 4. The EXFOR search window, including retrievals on target, reaction, reference and institutes, but also almost all keywords used in the EXFOR format.

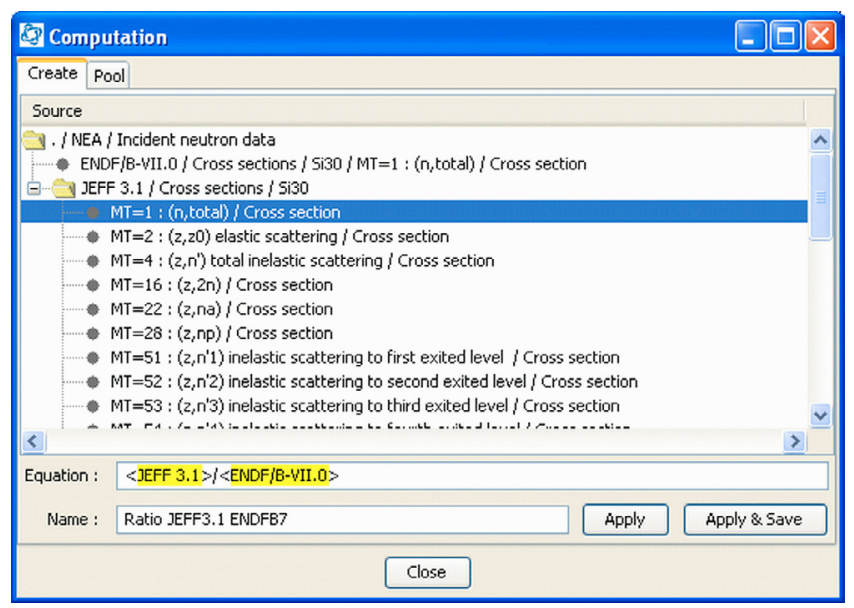

Fig. 5. The Computation dialog, where the calculation of the ratio between the JEFF-3.1 and ENDF/B-VII total cross section is being prepared.

\subsection{Computation of data}

JANIS was specially designed to offer flexibility for the comparison and computation of different data sets, as exemplified in figure 5, where the ratio of the total cross section in JEFF-3.1 and ENDF/B-VII is calculated. These features (such as linear combinations, ratios of data, flux weighting) were earlier restricted to cross-section data only. It is now possible to perform more advanced combinations such as a product of cross-sections with energy and angular distributions. The possibilities include point-wise cross-sections compared to weighted multi-group crosssections, or ratios of different reaction data. The results can easily be plotted or tabulated in the plot window. The equation editor window, offers the possibility to store and re-use equations.

\section{Future upgrades of the software}

JANIS has been enhanced thanks to users' feedbacks and comments which are always welcome. Users can comment 
errors via the JANIS bug report functionality or directly send suggestions by email to janisinfo@ nea.fr. All feedback is very important in any form.

For the next major release we plan to complete the coverage of ENDF format. This includes the possibility to display photo-nuclear data and covariance matrices. These kinds of data are of increasing importance in the field of nuclear application data [5].

For many evaluators a key functionality would be to verify the data in the original format, why the raw data formats are to be available as well as output formats. Another issue often expressed is the need of a simple tool for mixing natural elements from microscopic isotopic data. This will be developed within the frame of the general computational tool.

Links between ENDF libraries and EXFOR will be improved, to facilitate comparison between these datasets, EXFOR is a very flexible format so conversions are required to compare some data with their ENDF counterpart, like units conversion or even variable conversion (e.g., for angular distribution which are given as function of cosine of angle in ENDF libraries and either cosine of angle or angle in EXFOR).

Integration with the NEA retrieval web pages can go further, the goal being to take profit of JANIS's navigation and display functionalities in our database web retrieval pages, with the minimum requirements on the client side (even without Java installed). This will be an additional way of using JANIS; existing ones will continue to be maintained (standalone application launched from the DVD or installed on hard-drive, Java Web Start application launched from the web page).

Naturally feedback and requests for changes and modifications are welcomed. We would like to thanks all users who have sent bug reports or comments over the years. This is very helpful for us.

\section{References}

1. A. Hasegawa et al., The nuclear data activities at the NEA Data Bank (these proceedings).

2. H. Henriksson et al., The art of collection experimental data internationally: EXFOR, CINDA and the NRDC network (these proceedings).

3. M. Herman (Ed.), ENDF-102, ENDF-6 data formats and procedures for the evaluated nuclear data file ENDF-VII, BNL-NCS 44945-01/04-Rev (2005).

4. G. Audi et al., Nucl. Phys. A 729, 3 (2003) .

5. A. Koning et al., Status and future work of the NEA working party on international nuclear data evaluation cooperation (these proceedings). 